\title{
Sampled-data Observer-based Glucose Control for the Artificial Pancreas
}

\author{
Alessandro Borri ${ }^{1}$, Pasquale Palumbo ${ }^{1}$, Costanzo Manes ${ }^{2}$, \\ Simona Panunzi ${ }^{1}$, Andrea De Gaetano ${ }^{1}$ \\ ${ }^{1}$ CNR-IASI Biomathematics Laboratory, Italian National Research Council (CNR), \\ 00168 Rome, Italy \\ 2 DISIM Department, University of L'Aquila, 67100 L'Aquila, Italy \\ \{alessandro.borri, simona.panunzi, andrea.degaetano\}@biomatematica.it, \\ pasquale.palumbo@iasi.cnr.it, costanzo.manes@univaq.it
}

\begin{abstract}
Artificial Pancreas (AP) is an expression referred to a set of techniques for the closed-loop control of the plasma glucose concentration by means of exogenous insulin administration in diabetic patients. Diabetes comprises a group of metabolic disorders characterized by high blood sugar levels over a prolonged period, due to pancreas failure to produce enough insulin and/or insulin resistance, so that higher amounts of insulin are usually required in order to keep glycemia in a safe range. In this work, we face the problem of glucose control for a class of Type-2 diabetic patients, in the presence of sampled glucose measurements and without any information about the time course of insulinemia. A compact physiological model of the glucose-insulin system is reviewed, then an observer (based on this model) is designed to estimate the insulin trajectory from the glucose samples. Finally, a feedback control law (based on the reconstructed state) is designed to deliver exogenous intra-venous insulin to each individual. Simulations have been performed in-silico on models of virtual patients, whose parameters are tuned according to real data, and aim at validating the method in the presence of parameter variations and quantization errors.
\end{abstract}

Keywords: Diabetes, Artificial Pancreas, Glucose Control, Observers, Feedback Systems

\section{Introduction}

In the past twenty years, with the improvement in technology allowing both direct measurement of glucose concentrations in the interstitium (or signals strongly correlated with it) and the availability of miniaturized hormonal pumps with acceptable autonomy, weight and precision of delivery, the automatic, closed-loop control of glycemia has become a real possibility. Together with the opening of technological opportunities there has naturally been the emergence of the need for theoretical analysis of the control algorithms to be employed in the practical industrial applications. This heterogeneous collection of devices, techniques, technology and theory, 
is known under the umbrella term "Artificial Pancreas" (AP). The main approach (though not the only one) to the regulation of the levels of glucose in blood has been the administration of carefully titrated amounts of the hormone insulin [1-3], which is notably completely lacking in Type-1 Diabetes Mellitus (T1DM) patients. Insulin promotes the uptake of glucose by peripheral tissues (particularly muscle and adipose tissue) and inhibits the release of autonomously synthesized glucose (from glycogen or other precursors) in the liver and kidney. Insulin is naturally formed in pancreatic beta cells, which are destroyed by the autoimmune processes typically characterizing the development of T1DM. To this lack of endogenous insulin, traditional medical therapy supplies with the administration of human or human-like hormone intravenously (IV) or subcutaneously (SC). The relevance of this topic is determined by the fact that T1DM affects approximately the $1 \%$ of the world population, with a huge impact on health expenditure by industrialized countries. While not so dramatic in its onset as T1DM, adult-onset or Type-2 Diabetes Mellitus (T2DM) also represents a huge burden on the health system due to the fact that its prevalence is not only vastly greater than that of T1DM (about 10 times as frequent), but also that T2DM incidence is increasing, to epidemic proportions, due to the spreading of excessively rich dietary habits from western to emerging Countries. In T2DM, the original defect consists in a lack of effect of insulin ("insulin resistance"): the hormone is initially secreted in higher than normal amounts by the pancreas, in an attempt to correct hyperglycemia resulting from insulin resistance. With the progression of disease, however, glucose toxicity and possibly other factors determine first a relative, then an absolute deficiency of insulin secretion, with an accelerated worsening of the individual conditions and the development of the clinical picture of frank diabetes mellitus. In this situation, the patient undergoes a progressive step-up of the therapeutic measures employed, going from simple dieting and increase in physical exercise, to oral hypoglycemic agents of different kinds to supplemental insulin therapy.

In this framework, the theory on the artificial control of glycemia has had to address a number of problems, stemming from the nonlinear and delayed insulin response [4,5], the availability of observations on glucose only, and the high variability of the insulin determinations that can be obtained with radio-immunological methods [6]. One fruitful way to address these problems has been through the shift from modelless to model-based control algorithms, in which the controller is synthesized using the model equations themselves. It is clear that, in this procedure, the smaller, the more general, the easier to implement, and the more robust the physiological model is, the better the resulting characteristics of the controller will be. It is clear therefore that the physiological model used to interpret the data and realize the controller must be relatively small and have easily identifiable parameters; it must, in other words, be a "compact" model [14], possibly even allowing to find an analytical solution to the control problem. In order to validate the controller based on the compact model, however, some "extended" model of the same physiological system must be used, more realistic, with parameters taken from the literature or decided upon by physicians to represent the kind of patients under investigation. In this way, the possible control strategies can be directly simulated and tested in silico.

We will use as a compact model a Delay-Differential Equations (DDE) model we 
have previously published $[7,8]$, which has been demonstrated to exhibit much better properties than alternative "minimal" models [13], and which we have already used in several different situations [9-12]. It must be noticed that the use of DDEbased glycemia-control algorithms can be equally well applied to both T1DM and T2DM patients, where in the latter case pancreatic Insulin Delivery Rate (IDR) also needs to be modelled, and in which IDR exhibits random variability [5]. Previous work published on this DDE model include having demonstrated that it can be used to safely control glycemia down to normal levels in T2DM subjects [9] and having validated observer-based controls against a widely known extended model [10], while current research effort is being dedicated to new therapeutic insulin dosing approaches for T2DM patients [16].

The goal of the present work is to consider the problem of controlling glycemia based upon sampled measurements. Unlike most of the contributions having appeared in the literature so far, we will assume not only non-availability of serum insulin determinations, but also the availability of glycemia measurements only at discrete sampling times, as it happens in the Continuous Glucose Monitoring (CGM) [17] technique of patient surveillance, which is the motivating reason of the present analysis. The observer we will use is constructed as shown in Cacace et al. $[18,19]$. In contrast with previous work [9], we will therefore not assume glycemia to be measurable over continuous time. Strictly speaking, Cacace's construction cannot be applied when the compact model is delayed, so we limit our analysis to the situation in which the delay in insulin response is small (shorter than one minute). The control algorithm, based on the estimated state, will deliver exogenous intra-venous insulin continuously, with changes in insulin administration rate happening at sampling times. In order to make our simulations more realistic, we introduce further real-life complications, such as quantization (modeling the possible lack of accuracy of the instrument as well as the analog-to-digital and digital-to-analog conversion processes) both in the measurement and in the control phases.

The paper is structured as follows: in Section 2, we review some theoretical results about observer-based closed-loop control methods; in Section 3, we describe a model of the glucose-insulin system in terms of ordinary differential equations; in Section 4, we apply the methods described in Section 2 to the glucose-insulin model to find a control strategy (in terms of exogenous insulin rate) aiming at tracking desired glucose trajectories; Section 5 illustrates preliminary in-silico validation results for the described framework, obtained in an experimental setup utilizing data coming from real patients. Some final remarks and comments on future work conclude the article. 


\section{Review of Observer-based Closed-Loop Control Design}

Consider a system of nonlinear differential equations in the form

$\left\{\begin{aligned} \dot{x}(t) & =f(x(t))+g(x(t)) u(t), & & t \geq 0 \\ y(t) & =c(x(t-\delta(t))), & & t \geq \Delta\end{aligned}\right.$

where $x(t) \in \mathbb{R}^{n}$ denotes the state vector, $\dot{x}(t):=\frac{d x(t)}{d t}$ is its time derivative, $u(t) \in \mathbb{R}$ is the input function, $y(t) \in \mathbb{R}$ is the measured output, $\delta(t) \in[0, \Delta]$ is the output time-varying measurement delay (known), $x_{0} \in \mathbb{R}^{n}$ is the initial state, $g(x)$ and $f(x)$ are $C^{\infty}$ vector fields and $c(x)$ is a $C^{\infty}$ function.

The problem of asymptotic state observation consists in the design of a causal system producing a vector variable $\hat{x}(t)$, which is called observed state, asymptotically converging to the real state $x(t)$ (i.e., $\|x(t)-\hat{x}(t)\| \rightarrow 0$ ), from the knowledge of the pair $(u(t), y(t))$. Such a system is called an asymptotic observer; additionally, it is said to be an exponential observer if there exist $\mu>0$ and $\alpha>0$ such that

$\|x(t)-\hat{x}(t)\| \leq \mu e^{-\alpha t}\|x(0)-\hat{x}(0)\|$,

for any $x(0)$ and $\hat{x}(0)$ in $\mathbb{R}^{n}$.

With the aim of designing such an observer, we first define the drift-observability map $z=\phi(x)$, stacking the first $n$ Lie derivatives (from 0 to $n-1$ ) of the output function $c(x)$ along the drift vector field $f(x)$, and its Jacobian $Q(x)$, as

$z=\left[\begin{array}{c}z_{1} \\ z_{2} \\ \vdots \\ z_{n}\end{array}\right]=\phi(x):=\left[\begin{array}{c}h(x) \\ L_{f} c(x) \\ \vdots \\ L_{f}^{n-1} c(x)\end{array}\right], \quad Q(x):=\frac{\partial \phi(x)}{\partial x}$.

The observer in [18, 19], also reviewed in [20], takes the following expression:

$\dot{\hat{x}}(t)=f(\hat{x}(t))+g(\hat{x}(t)) u(t)+e^{-\eta \delta(t)} Q^{-1}(\hat{x}(t)) K\{y(t)-c(\hat{x}(t-\delta(t)))\}$,

where the gain matrix $K$ assigns the $n$ eigenvalues of $(A-K C)$ so that the estimation error $x(t)-\hat{x}(t)$ asymptotically vanishes, with

$A:=\left[\begin{array}{cc}0_{(n-1) \times 1} & I_{(n-1) \times(n-1)} \\ 0 & 0_{1 \times(n-1)}\end{array}\right], \quad C \quad:=\left[\begin{array}{ll}1 & 0_{1 \times(n-1)}\end{array}\right]$,

and $\eta>0$ is a design parameter, whose role is to assign a larger weight to the more recent measurements with respect to the older ones.

Under some technical hypotheses (including, in particular, uniform Lipschitz driftobservability and uniform input boundedness), if the system has full relative degree, it is possible to demonstrate the following theorem, establishing the exponential convergence to zero of the observation error. 
Theorem 1. [19] Given the system (1), with $\delta(t) \in[0, \Delta]$, for any assigned $\eta>0$, there exists $K$ and a positive $\bar{\Delta}$ such that the system in (4) is a global exponential observer for the system in (1), provided that $\Delta<\bar{\Delta}$, with $\eta$ being the estimation error decay rate (namely, (2) holds with $\alpha=\eta$ and some $\mu>0$ ).

We remark that the previous result allows to employ the observer (4) if the sampling interval is smaller than or equal to $\Delta$. If this is not the case, as shown in [19], a chain of sampled observers can be built.

In order to close the control loop, an input-output linearization approach is adopted, assuming that the relative degree of the system is $n$ (see, e.g., [22]). The observability map dynamics in (3) rewrites:

$\dot{z}=\frac{\partial \phi(x)}{\partial x} \dot{x}=Q(x)(f(x)+g(x) u)$.

We impose the virtual input $v:=\dot{z}_{n}=L_{f}^{n} c(x)+L_{g} L_{f}^{n-1} c(x) u$, in order to obtain the linearizing feedback law:

$u=\frac{v-L_{f}^{n} c(x)}{L_{g} L_{f}^{n-1} c(x)}$.

The virtual input $v$ needs to be chosen with the aim of tracking desired trajectories for the closed-loop system. To this end, a smooth reference output signal $y_{\text {ref }}(t)$ is defined, along with the vector of its first $n$ time derivatives

$z_{r e f}(t)=\left[\begin{array}{c}z_{1, r e f}(t) \\ z_{2, r e f}(t) \\ \vdots \\ z_{n, r e f}(t)\end{array}\right]=\left[\begin{array}{c}y_{r e f}(t) \\ \dot{y}_{r e f}(t) \\ \vdots \\ y_{r e f}^{(n-1)}(t)\end{array}\right]$,

and defining $e:=z-z_{\text {ref }}$, the error equation is

$\dot{e}=A e+B\left(v-\dot{z}_{n, r e f}\right), \quad$ with $\quad B:=\left[\begin{array}{c}0_{(n-1) \times 1} \\ 1\end{array}\right]$.

Since the Brunovsky pair $(A, B)$ is reachable, it is sufficient to set

$v=H e+\dot{z}_{n, r e f}$

to guarantee the exponential convergence to zero of the linearized error dynamics, with rate determined by the $n$ eigenvalues of matrix $(A+B H)$, assigned by means of $H$.

As a final remark, we notice that the control law reported in Eq. (7)-(8) is a continuous state-feedback control strategy, which depends on the continuous state $x(t)$, which is usually not available, except for its estimate $\hat{x}(t)$ provided by the observer (4). So, it is possible to restate the control law in (7)-(8) in terms of a feedback 
from the reconstructed state, but this is not guaranteed to work, in general, in the non-linear case, although local convergence results exist in the literature. In the linear case, instead, the separation principle would guarantee the asymptotic convergence of the output $y(t)$ to its reference value $y_{\text {ref }}(t)$.

\section{A Continuous-Discrete Model of the Glucose-Insulin System}

Continuous-discrete models refer to physical continuous-time systems with measurements acquired at discrete sampling times. These models often appear in clinical/medical applications like those related to the Artificial Pancreas, with control design problems related to the lack of a continuous stream of output data. According to [19], discrete measurements can still be formalized by means of a continuoustime output function. To this end, for a sampling sequence $\left\{t_{i}\right\}$ and assuming to measure plasma glucose concentration $G\left(t_{i}\right)$, the piecewise-constant output function $y(t)$ defined as

$y(t)=G\left(t_{i}\right) \quad t \in\left[t_{i}, t_{i+1}\right), \quad i=0,1, \ldots$

can be restated as a delayed output in the equivalent form

$y(t)=G(t-\delta(t)) \quad t \geq 0$,

where the delay $\delta(t)$ within any two consecutive sampling instants is time-varying:

$\delta(t)=t-t_{i}, \quad t \in\left[t_{i}, t_{i+1}\right), \quad i=0,1, \ldots$,

with $t_{0}=0$. The sampling interval has a uniform upper bound equal to $\Delta:=$ $\max _{i}\left(t_{i+1}-t_{i}\right)$.

As shown in the previous section, this formal setting of the model output function allows to design exponential observers and observer-based control laws, which have been recently exploited also in the context of the artificial pancreas $[9,10,21]$. To this end, we consider a modified version of the DDE model presented in $[7,8]$ and exploited in $[9,10]$, which contains an explicit discrete delay modeling the secondary insulin released for varying plasma glucose concentration. Since we need to restate into the form of Eq. (1), the delay of the glucose-stimulated insulin production rate is neglected. This fact clearly limits the proposed feedback control law applicability and refers to further developments of the mathematical theory possibly including time-delay systems. Nonetheless, this work aims at showing the proof of concept of an observer-based control law in such continuous-discrete systems.

In absence of delay, the equations of model $[7,8]$ are particularized as follows:

$\begin{cases}\frac{d G(t)}{d t} & =-K_{x g i} G(t) I(t)+\frac{T_{g h}}{V_{G}}, \\ \frac{d I(t)}{d t} & =-K_{x i} I(t)+\frac{T_{i G \max }}{V_{I}} h(G(t))+u(t), \quad t \geq 0\end{cases}$

with initial conditions $G(0)=G_{0}, I(0)=I_{0}$, where: 
- $G(t)$ is the glucose concentration in the plasma at time $t[m M]$;

- $I(t)$ is the insulin concentration in the plasma at time $t[p M]$;

- $K_{x g i}$ is the rate of glucose uptake by tissues per unit of plasma insulinemia $\left[\min ^{-1} p M^{-1}\right]$;

- $T_{g h}$ is the net balance between hepatic glucose output and zero-order glucose tissue uptake $\left[\mathrm{min}^{-1}(\mathrm{mmol} / \mathrm{KgBW})\right]$;

- $V_{G}$ is the apparent distribution volume for glucose $[L / \mathrm{kgBW}]$;

- $K_{x i}$ is the apparent linear insulin clearance rate $\left[\mathrm{min}^{-1}\right]$;

- $T_{i G m a x}$ is the maximal second-phase insulin release rate $\left[\mathrm{min}^{-1}(\mathrm{pmol} / \mathrm{kgBW})\right]$;

- $V_{I}$ is the apparent insulin distribution volume $[\mathrm{L} / \mathrm{kgBW}]$;

- $h(\cdot)$ is a nonlinear function representing the endogenous pancreatic Insulin Delivery Rate (IDR) as

$$
h(G)=\frac{\left(G / G^{*}\right)^{\gamma}}{1+\left(G / G^{*}\right)^{\gamma}}
$$

where $\gamma$ (dimensionless) denotes the progressiveness of the pancreas reaction to circulating glucose concentrations and $G^{*}[\mathrm{mM}]$ is the glucose concentration at which the insulin release reaches half of its maximal rate;

- $u(t)$ is the exogenous intra-venous insulin delivery rate at time $t$, which takes the role of control input $[\mathrm{pM} / \mathrm{min}]$.

The model in (11) enjoys some interesting properties:

- it is statistically robust, in that its parameters are statistically identifiable with very good precision by means of standard perturbation experiments, such as the Intra-Venous Glucose Tolerance Test (IVGTT) [7, 13];

- it is a compact model, in the sense that according to a "minimal" set of independent parameters, it allows to very well resemble the physiology of the glucose/insulin kinetics [7];

- it is mathematically consistent, in that exhibits satisfactory properties of the solutions [8]; in particular: positivity, boundedness, and a unique positive stable equilibrium.

Identification issues and statistical robustness of this model are discussed in [7], whilst the work [8] exhaustively treats its structural properties and the qualitative behavior of its solutions.

\section{The Artificial Pancreas}

We now apply the control design methodology illustrated in Section 2 to the glucoseinsulin model described in Section 3. By restating in the vector form $x(t)=\left[x_{1}(t), x_{2}(t)\right]^{T}=$ 
$[G(t), I(t)]^{T}$ the already defined state variables, a compact expression in the form (1) is obtained for (10)-(11):

$$
\left\{\begin{array}{llll}
\dot{x}(t) & =f(x(t))+B u(t), & & t \geq 0 \\
y(t) & =C x(t-\delta(t)), & & t \geq \Delta \\
\delta(t) & =t-t_{i}, \quad t \in\left[t_{i}, t_{i+1}\right), & & i=0,1, \ldots
\end{array}\right.
$$

where

$$
\begin{aligned}
& f(x)=\left[\begin{array}{l}
f_{1}(x) \\
f_{2}(x)
\end{array}\right]=\left[\begin{array}{c}
-K_{x g i} x_{1} x_{2}+\frac{T_{g h}}{V_{G}} \\
-K_{x i} x_{2}+\frac{T_{i G \max }}{V_{I}} h\left(x_{1}\right)
\end{array}\right], \\
& \delta(t) \in[0, \Delta], \quad B=\left[\begin{array}{ll}
0 & 1
\end{array}\right]^{T}, \quad C=\left[\begin{array}{ll}
1 & 0
\end{array}\right] .
\end{aligned}
$$

The drift-observability map $z=\phi(x)$ and its Jacobian are

$$
\begin{aligned}
& z=\left[\begin{array}{l}
z_{1} \\
z_{2}
\end{array}\right]=\phi(x):=\left[\begin{array}{c}
C x \\
C f(x)
\end{array}\right]=\left[\begin{array}{c}
x_{1} \\
f_{1}(x)
\end{array}\right]=\left[\begin{array}{c}
x_{1} \\
-K_{x g i} x_{1} x_{2}+\frac{T_{g h}}{V_{G}}
\end{array}\right], \\
& Q(x):=\frac{\partial \phi(x)}{\partial x}=\left[\begin{array}{cc}
1 & 0 \\
-K_{x g i} x_{2} & -K_{x g i} x_{1}
\end{array}\right],
\end{aligned}
$$

where invertibility is guaranteed for $x_{1} \neq 0$.

The observer equation in (4) is

$\dot{\hat{x}}(t)=f(\hat{x}(t))+B u(t)+e^{-\eta \delta(t)} Q^{-1}(\hat{x}(t)) K\{y(t)-C \hat{x}(t-\delta(t))\}$,

where the eigenvalues $\lambda_{1}<0, \lambda_{2}<0$ of $(A-K C)$ are assigned by means of $K=$ $\left[\begin{array}{c}-\left(\lambda_{1}+\lambda_{2}\right) \\ \lambda_{1} \lambda_{2}\end{array}\right]$ with the aim of ensuring the exponential convergence to zero of the error $x(t)-\hat{x}(t)$, and where $A=\left[\begin{array}{ll}0 & 1 \\ 0 & 0\end{array}\right]$.

By explicitly rewriting $\hat{x}(t)=\left[\hat{x}_{1}(t), \hat{x}_{2}(t)\right]^{T}=[\hat{G}(t), \hat{I}(t)]^{T}$, for all times $t \in\left[t_{i}, t_{i+1}\right)$ and $i=0,1, \ldots$, the observer $(4)$ is component-wise rewritten as

$$
\left\{\begin{array}{l}
\frac{d \hat{G}(t)}{d t}=-K_{x g i} \hat{G}(t) \hat{I}(t)+\frac{T_{g h}}{V_{G}}+e^{-\eta \delta(t)}\left(\lambda_{1}+\lambda_{2}\right)\left(G\left(t_{i}\right)-\hat{G}\left(t_{i}\right)\right), \\
\frac{d \hat{I}(t)}{d t}=-K_{x i} \hat{I}(t)+\frac{T_{i G \max }}{V_{I}} h(\hat{G}(t))+u(t)+e^{-\eta \delta(t)} \frac{K_{x g i}\left(\lambda_{1}+\lambda_{2}\right) \hat{I}(t)-\lambda_{1} \lambda_{2}}{K_{x g i} \hat{G}(t)}\left(G\left(t_{i}\right)-\hat{G}\left(t_{i}\right)\right) .
\end{array}\right.
$$

The technical assumptions of Theorem 1 are fulfilled for the glucose-insulin system in (10)-(11), which ensures that the observation error exponentially vanishes. 
We now detail the algorithm of glucose control. The observability map evolution in (13) rewrites:

$$
\begin{aligned}
\dot{z} & =\left[\begin{array}{c}
\dot{z}_{1} \\
\dot{z}_{2}
\end{array}\right]=\frac{\partial \phi(x)}{\partial x} \dot{x}=Q(x)(f(x)+B u)=\left[\begin{array}{cc}
1 & 0 \\
-K_{x g i} x_{2} & -K_{x g i} x_{1}
\end{array}\right]\left[\begin{array}{c}
f_{1}(x) \\
f_{2}(x)+u
\end{array}\right] \\
& =\left[\begin{array}{cc}
1 & 0 \\
-K_{x g i} x_{2} & -K_{x g i} x_{1}
\end{array}\right]\left[\begin{array}{c}
z_{2} \\
-K_{x i} x_{2}+\frac{T_{i G \max }}{V_{I}} h\left(x_{1}\right)+u
\end{array}\right],
\end{aligned}
$$

so one obtains

$$
\left\{\begin{array}{l}
\dot{z}_{1}=z_{2}, \\
\dot{z}_{2}=-K_{x g i} x_{2}\left(-K_{x g i} x_{1} x_{2}+\frac{T_{g h}}{V_{G}}\right)+K_{x g i} x_{1}\left(K_{x i} x_{2}-\frac{T_{i G \max }}{V_{I}} h\left(x_{1}\right)\right)-K_{x g i} x_{1} u .
\end{array}\right.
$$

We now get the linearizing feedback law by setting $\dot{z}_{2}:=v$ to obtain

$u=K_{x i} x_{2}-\frac{T_{i G \max }}{V_{I}} h\left(x_{1}\right)-\frac{v+K_{x g i} x_{2}\left(-K_{x g i} x_{1} x_{2}+\frac{T_{g h}}{V_{G}}\right)}{K_{x g i} x_{1}}$

which is computable for positive glycemias $x_{1}$, in agreement with the Jacobian matrix $Q(x)$ in (14) being invertible.

The reference glycemia trajectory is

$y_{\text {ref }}(t)=G_{r e f}(t)=G_{d}+\left(G_{b}-G_{d}\right) e^{-\lambda t}$,

with $\lambda>0$, and its goal is to lead the glycemia of an individual from a high basal value $G_{b}$ of a subject to a lower healthier value $G_{d}$. By defining

$z_{\text {ref }}=\left[\begin{array}{c}z_{1, r e f} \\ z_{2, \text { ref }}\end{array}\right]:=\left[\begin{array}{l}y_{\text {ref }} \\ \dot{y}_{\text {ref }}\end{array}\right]$,

its dynamics is readily computed:

$\dot{z}_{\text {ref }}(t)=\left[\begin{array}{l}\dot{z}_{1, r e f}(t) \\ \dot{z}_{2, r e f}(t)\end{array}\right]=\left[\begin{array}{c}z_{2, r e f}(t) \\ \dot{z}_{2, r e f}(t)\end{array}\right]=\left[\begin{array}{c}-\lambda\left(G_{b}-G_{d}\right) e^{-\lambda t} \\ \lambda^{2}\left(G_{b}-G_{d}\right) e^{-\lambda t}\end{array}\right]$.

The error $e:=z-z_{\text {ref }}$ is described by the equation

$\dot{e}=\left[\begin{array}{c}\dot{z}_{1}-\dot{z}_{1, r e f} \\ \dot{z}_{2}-\dot{z}_{2, r e f}\end{array}\right]=\left[\begin{array}{c}z_{2}-z_{2, r e f} \\ v-\dot{z}_{2, r e f}\end{array}\right]=A e+B\left(v-\dot{z}_{2, \text { ref }}\right)$.

Finally, we assign

$v=H e+\dot{z}_{2, r e f}$

to guarantee the convergence to zero of the linearized error dynamics, whose convergence rate is determined by the eigenvalues $\lambda_{3}<0, \lambda_{4}<0$ of matrix $(A+B H)$, assigned by $H=\left[\begin{array}{c}-\lambda_{3} \lambda_{4} \\ \left(\lambda_{3}+\lambda_{4}\right)\end{array}\right]^{T}$. 
As discussed at the end of Section 2, in the spirit of separation principle, we restate Eqs. (19)-(20) in terms of a control from the estimated state, leading to the following continuous feedback law

$u=\max \left\{0, K_{x i} \hat{x}_{2}-\frac{T_{i G m a x}}{V_{I}} h\left(\hat{x}_{1}\right)-\frac{H\left(\hat{z}-z_{r e f}\right)+\dot{z}_{2, r e f}+K_{x g i} \hat{x}_{2}\left(-K_{x g i} \hat{x}_{1} \hat{x}_{2}+\frac{T_{g h}}{V_{G}}\right)}{K_{x g i} \hat{x}_{1}}\right\}$

where $\hat{z}:=\left[\begin{array}{c}\hat{x}_{1} \\ f_{1}(\hat{x})\end{array}\right]$, and $\hat{x}=\left[\begin{array}{c}\hat{x}_{1} \\ \hat{x}_{2}\end{array}\right]=\left[\begin{array}{c}\hat{G} \\ \hat{I}\end{array}\right]$ is the output of the observer in (16). We remark that the possibility of a negative exogenous insulin rate in (21) is formally inhibited.

\section{In-silico Evaluation}

We here evaluate the performance of the techniques illustrated in the previous sections in a non-ideal experimental context. We start from the data obtained from 3 healthy subjects, whose samples of glucose and insulin are included in the data collected in [7]. Some anthropometric data for these subjects are summarized in Table 1. Each individual underwent an Intra-Venous Glucose Tolerance Test (IVGTT), according to which a glucose bolus is administered intra-venously after an overnight fasting period, and then plasma glucose and serum insulin concentration are sampled for the following 3 hours, at varying sampling time. IVGTT is also considered among the most affordable and commonly used perturbation procedures used to estimate insulin sensitivity. Measurements of glycemia and insulinemia from this experiment are used to identify the parameters of the ODE model (11), which is coincident with the DDE model in [7], [8] in the particular case $\tau_{g}=0$. As a matter of fact, as already mentioned before, just subjects with negligible delay in the glucose action on pancreatic IDR are considered, following the sample-based approach in [19], and in absence of theoretical results for this method when applied to systems expressed by delayed differential equations.

After the identification phase, since some of the considered subjects are pre-diabetic and not diabetic, we artificially perturbate the parameters in order to simulate a potential natural progression of the disease towards diabetes (see also [9]). In particular, we reduced the insulin resistance (up to about $K_{x g i}<10^{-4}$ ) and the pancreatic glucose sensitivity $T_{i G \max }$, to then recompute some of the other parameters via the algebraic steady-state conditions obtained from the model in Eq. (11). In more details, the basal values of glycemia $G_{b}$ and insulinemia $I_{b}$, representing the equilibria of (11) in absence of exogenous insulin administration $(u=0)$, are obtained from:

$\begin{cases}K_{x g i} V_{G} G_{b} I_{b} & =T_{g h}, \\ K_{x i} V_{I} I_{b} & =T_{i G \max } h\left(G_{b}\right) .\end{cases}$

Table 2 collects the parameter values for the three individuals. Note that the parameters of each model are assumed to be known (up to some uncertainty) in the 
Table 1

Numerical values of some anthropometric parameters (in the respective units of measurement) for the 3 patients considered.

\begin{tabular}{|c|c|c|c|}
\hline Parameter & Patient 1 & Patient 2 & Patient 3 \\
\hline Sex & Male & Female & Female \\
\hline Age [years] & 32 & 26 & 27 \\
\hline Height $[\mathrm{m}]$ & 1.69 & 1.57 & 1.56 \\
\hline Body Weight $[\mathrm{kg}]$ & 68 & 48 & 57 \\
\hline Body Mass Index $\left[\mathrm{kg} / \mathrm{m}^{2}\right]$ & 23.81 & 19.47 & 23.42 \\
\hline
\end{tabular}

Table 2

Model parameters values (and units of measurement) used in the in-silico evaluation.

\begin{tabular}{|c|c|c|c|}
\hline Parameter & Patient 1 & Patient 2 & Patient 3 \\
\hline$G_{b}$ & 8.96 & 8.78 & 8.44 \\
\hline$I_{b}$ & 27.82 & 24.04 & 7.04 \\
\hline$K_{x g i}$ & $7.45 \cdot 10^{-5}$ & $9.96 \cdot 10^{-5}$ & $5.39 \cdot 10^{-5}$ \\
\hline$T_{g h}$ & 0.0025 & 0.0027 & 0.0003 \\
\hline$V_{G}$ & 0.13 & 0.13 & 0.10 \\
\hline$K_{x i}$ & 0.10 & 0.06 & 0.25 \\
\hline$T_{i G \max }$ & 1.39 & 0.75 & 0.94 \\
\hline$V_{I}$ & 0.24 & 0.25 & 0.25 \\
\hline$\gamma$ & 2.30 & 2.52 & 1.52 \\
\hline$G^{*}$ & 9 & 9 & 9 \\
\hline
\end{tabular}

construction of the artificial pancreas tailored to the particular patient, in the spirit of the so-called personalized medicine approach.

In addition to the hypotheses dealt with in the theoretical part, we consider a more realistic simulation setting and assume a quantization error both in the measuring and in the control procedure, accounting for the processes of analog-to-digital and digital-to-analog conversion in digital devices. Quantization steps of $0.1 \mathrm{mM}$ for the glycemia measurements and $20 \mathrm{pM} / \mathrm{min}$ for the exogenous Insulin Delivery Rate (IDR) are assumed, respectively. Accordingly, quantization errors affect the initial values of the observer-based controller. The sampling time of the glycemia measurements is assumed constant and equal to $t_{i+1}-t_{i}=\Delta$, for all observations $i$, so that we can write more simply $t_{i}=i \cdot \Delta$, with $\Delta=5$ [min], which is a typical value for many Continuous-Glucose-Monitoring (CGM) devices currently available on the market [23]. We also assume that control samples are held for the same interval, without any phase shifts.

The Artificial Pancreas is designed by considering the individual parameters for each patient in Table 2, but an additional random uncertainty (up to $\pm 5 \%$ ) is considered with respect to the real values. The parameter $\eta$ in (16) is set equal to 5, the target glycemia is equal to $G_{d}=5 \mathrm{mM}$, the decay rate is $\lambda=1 / 30$. The same closed-loop eigenvalues for all patients are set: $\lambda_{1}=-0.8, \lambda_{2}=-1.6, \lambda_{3}=-1$, 

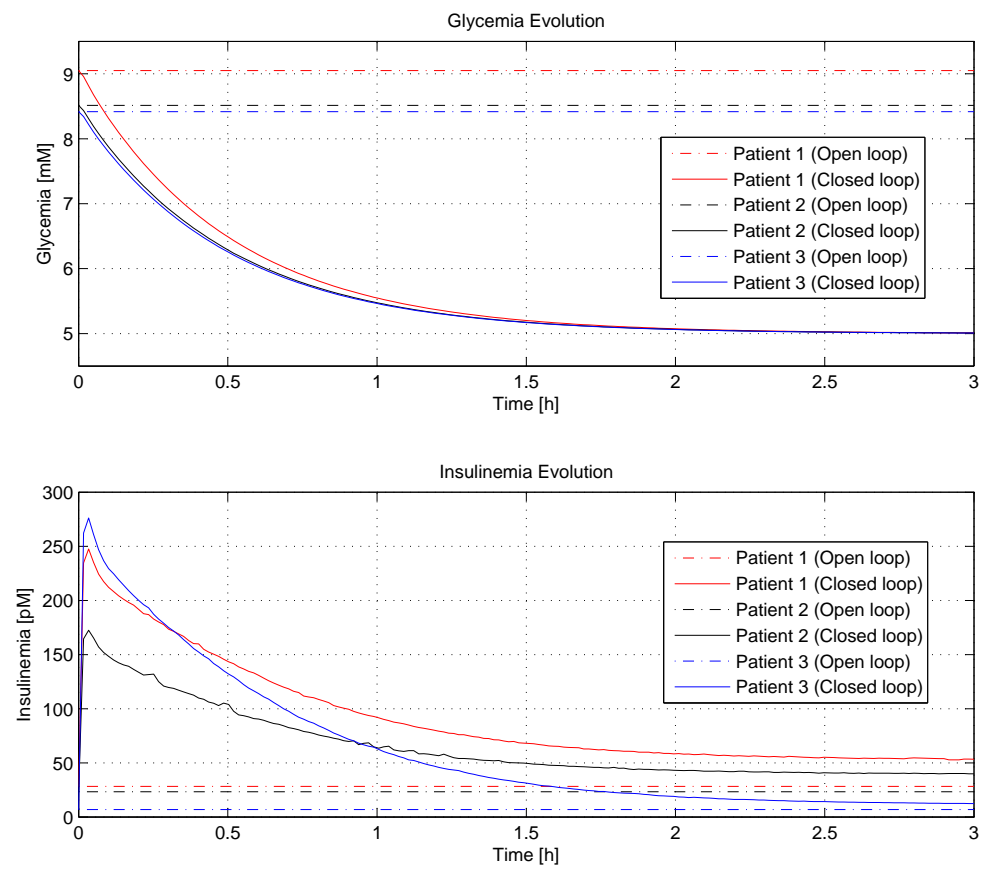

Figure 1

Top panel: trajectories of glycemia for 3 virtual patients: basal values (dash-dotted lines) and patients controlled by means of the Artificial Pancreas (solid lines).

Bottom panel: trajectories of insulinemia for 3 virtual patients: basal values (dash-dotted lines) and patients controlled by means of the Artificial Pancreas (solid lines).

$\lambda_{4}=-0.5$, uniquely determining the values of the observer gain $K$ in (4) and the control gain $H$ in (21).

Figures 1 and 2 illustrate the results in terms of glycemia and insulinemia trajectories, glucose percent error and IDR input. We note that the glucose trajectories (Fig. 1, top panel) monotonically decrease towards the target value $G_{d}$, which is reached, in all the subjects, within the experiment time horizon (3 hours). Correspondingly, the insulinemia trajectory (Fig. 1, bottom panel) shows an initial peak (exceeding $150 \mathrm{pM}$ for the three patients), to then recover towards levels below the 50- $p M$ value. Higher values of insulinemia (patient 3) correspond to higher exogenous insulin infusions (Fig. 2, bottom panel). In spite of the different parameters and initial conditions, the error falls below $10 \%$ (with respect to the target glycemia $G_{d}$ ) within about 1 hour for all the patients (Fig. 2, top panel), due to the common choice of the closed-loop eigenvalues. 

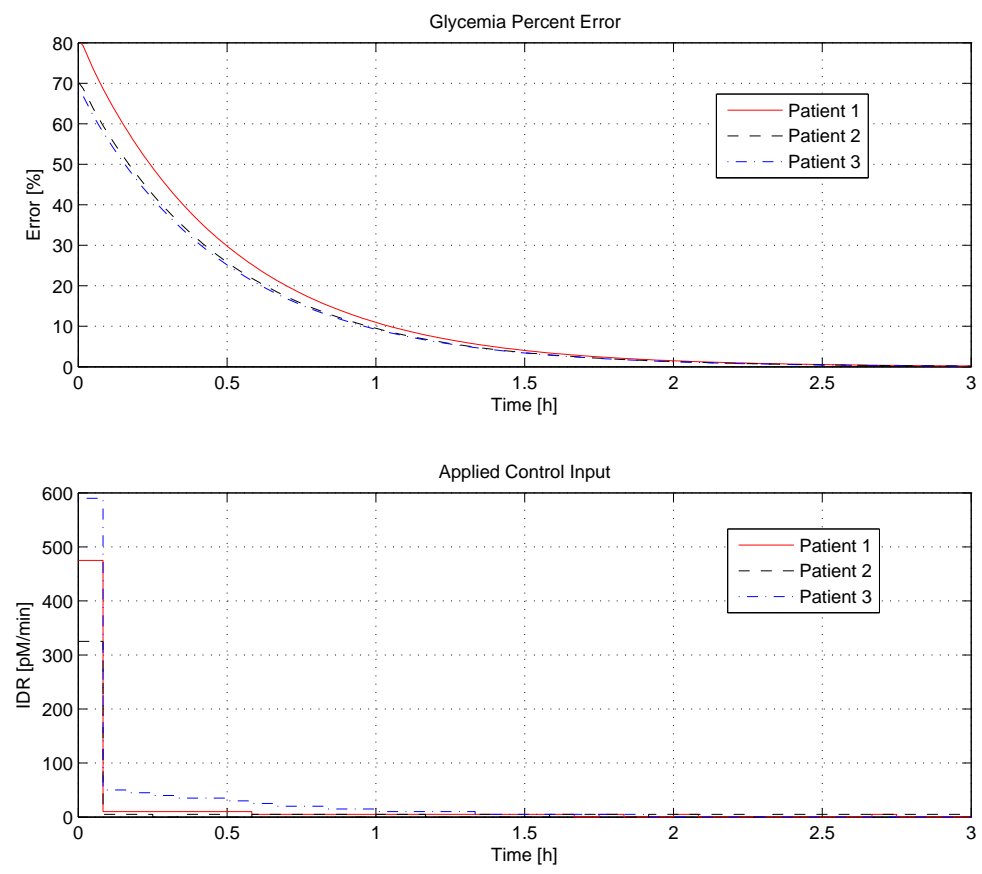

Figure 2

Top panel: glycemia percent error for the 3 virtual patients. Bottom panel: exogenous IDR input for the 3 virtual patients. 


\section{Discussion and Further Work}

In this work, we proposed a solution to a glucose control problem with partial/inaccurate information, in the direction of the development of the so-called Artificial Pancreas. After a general review of nonlinear output-feedback techniques, we considered a compact existing model constituted by nonlinear ordinary differential equations, which is known to represent adequately the evolution of the glucose-insulin system in people in which the apparent delay in the pancreatic second-phase insulin secretion can be approximately neglected. In this context, we designed an observer, which estimates the continuous dynamics of glucose and insulin from sparse measurements of glycemia. Then, the loop was closed by designing a feedback law from the observer state, and actuated in terms of exogenous insulin delivery, with the goal of tracking a proper trajectory of glycemia. A preliminary in-silico evaluation of the proposed methods has been performed on virtual patients whose parameters have been computed starting from real data, in a non-ideal simulation setup including quantization and parameter variations. The obtained results highlight that the approach can constitute a promising tool for studying and realizing an Artificial Pancreas in more realistic scenarios. In view of this goal, research studies will focus in the future on the validation of the techniques illustrated in this paper in the context of more comprehensive models (such as [15]), to better understand the way a real patient would react to the proposed treatment. In addition, formal extensions of the observer-based control to more general cases (state delays, discretized input and output) are under investigation.

\section{References}

[1] S.A. Weinzimer, G.M. Steil, K.L. Swan, J. Dziura, N. Kurtz, W.V. Tamborlane, Fully automated closed-loop insulin delivery versus semiautomated hybrid control in pediatric patients with type 1 diabetes using an artificial pancreas, Diabetes care, 31(5), 934-939, 2008.

[2] A.M. Albisser, B.S. Leibel, T.G. Ewart, Z. Davidovac, C.K. Botz, W. Zingg, H. Schipper, R. Gander, Clinical control of diabetes by the artificial pancreas, Diabetes, 23(5), 397-404, 1974.

[3] K. Zarkogianni, E. Litsa, K. Mitsis, P.-Y. Wu, C.D. Kaddi, C.-W. Cheng, M.D. Wang, K.S. Nikita, A Review of Emerging Technologies for the Management of Diabetes Mellitus, IEEE Trans. Biomed. Eng., 62(12), 2735-2749, 2015.

[4] A. Makroglou, J. Li, and Y. Kuang, Mathematical models and software tools for the glucose-insulin regulatory system and diabetes: An overview, Appl. Numer. Math., 56, 559-573, 2006.

[5] P. Palumbo, S. Ditlevsen, A. Bertuzzi, and A. De Gaetano, Mathematical modeling of the glucose-insulin system: A review, Math. Biosci., 244, 69-81, 2013.

[6] P. Palumbo, P. Pepe, S. Panunzi, and A. De Gaetano, Recent results on glucoseinsulin predictions by means of a state observer for time-delay systems, "Predic- 
tion Methods for Blood Glucose Concentration: Design, Use and Evaluation", H. Kirchsteiger, J. Jørgensen, E. Renard and L. Del Re Editors, Springer, 2015.

[7] S. Panunzi, P. Palumbo, and A. De Gaetano, A discrete single delay model for the intra-venous glucose tolerance test, Theor. Biol. Med. Model., 4, 2007.

[8] P. Palumbo, S. Panunzi, and A. De Gaetano, Qualitative behavior of a family of delay-differential models of the glucose-insulin system, Discrete Contin. Dyn. Syst. Ser. B., 7(2), 399-424, 2007.

[9] P. Palumbo, P. Pepe, S. Panunzi, and A. De Gaetano, Time-Delay Model-Based Control of the Glucose-Insulin System, by Means of a State Observer, Eur. J. Control, 6, 591-606, 2012.

[10] P. Palumbo, G. Pizzichelli, S. Panunzi, P. Pepe, and A. De Gaetano, Modelbased control of plasma glycemia: Tests on populations of virtual patients, Math. Biosci., 257, 2-10, 2014.

[11] P. Palumbo, P. Pepe, S. Panunzi, A. De Gaetano, Closed-loop glucose control: application to the Euglycemic Hyperinsulinemic Clamp, in Proc. 52nd Conf. Decision and Control, Dec. 2013, 4461-4466.

[12] P. Palumbo, G. Pizzichelli, P. Pepe, S. Panunzi, A. De Gaetano, Closed-loop control scheme for the Euglycemic Hyperinsulinemic Clamp: validation on virtual patients, in Proc. 19th IFAC World Congress of Automatic Control, 20882093, Cape Town, South Africa, 2014.

[13] S. Panunzi, A. De Gaetano, G. Mingrone, G., Advantages of the single delay model for the assessment of insulin sensitivity from the intravenous glucose tolerance test., Theoretical Biology \& Medical Modelling, 7, 9, 2010.

[14] F. Chee, T. Fernando, Closed loop control of blood glucose, Berlin, Heidelberg, Springer-Verlag, 2007.

[15] C. Dalla Man, R. Rizza, and C. Cobelli, Meal simulation model of the glucose insulin system IEEE Transactions on Biomedical Engineering, 54(10), 17401749, 2007.

[16] C.R. Gaz, A. De Gaetano, C. Manes, P. Palumbo, A. Borri, S. Panunzi, Effective Control of Glycemia using a Simple Discrete-delay Model, IFAC World Congress 2017, Toulouse, France, 2017 (to appear).

[17] W.V. Tamborlane, R.W. Beck, B.W. Bode, B. Buckingham, H.P. Chase, R. Clemons, et al., Continuous glucose monitoring and intensive treatment of type 1 diabetes, The New England journal of medicine, 359(14), 1464-1476, 2008.

[18] F. Cacace, A. Germani, and C. Manes, An observer for a class of nonlinear systems with time varying observation delay, Syst. Control Lett., 59, 305-312, 2010.

[19] F. Cacace, A. Germani, and C. Manes, A chain observer for nonlinear systems with multiple time-varying measurement delays, SIAM J. Control and Optim., 52.3, 1862-1885, 2014. 
[20] A. Borri, F. Cacace, A. De Gaetano, A. Germani, C. Manes, P. Palumbo, S. Panunzi, P. Pepe, Luenberger-like Observers for Nonlinear Time-Delay Systems with Application to the Artificial Pancreas, IEEE Control Systems Magazine, 2017 (conditionally accepted).

[21] L. Kovács, B. Pálancz, B. Zoltán, Design of Luenberger Observer for GlucoseInsulin Control via Mathematica, Proceedings of the 29th Annual International Conference of the 28th IEEE EMBS, 624-627, 2007.

[22] A. Isidori, Nonlinear control systems, Springer Science \& Business Media, 1995.

[23] D.B. Keenan, J.J. Mastrototaro, G. Voskanyan, G.M. Steil, Delays in Minimally Invasive Continuous Glucose Monitoring Devices: A Review of Current Technology, Journal of diabetes science and technology, 3.5, 1207-1214, 2009. 